\title{
Comercialização de Madeira de Florestas Naturais no Estado de Mato Grosso no Período de 2004 a 2009
}

\author{
Edilene Silva Ribeiro ${ }^{1}$, Roberto Antonio Ticle de Melo e Sousa ${ }^{2}$, \\ Marcelo Dias de Souza ${ }^{1}$, Alberto Dorval ${ }^{2}$, Reginaldo Brito da Costa ${ }^{2}$ \\ ${ }^{1}$ Programa de Pós-Graduação em Ciências Florestais e Ambientais, Faculdade de Engenharia Florestal - FENF, \\ Universidade Federal de Mato Grosso - UFMT \\ ${ }^{2}$ Departamento de Engenharia Florestal, Faculdade de Engenharia Florestal - FENF, \\ Universidade Federal de Mato Grosso - UFMT
}

\begin{abstract}
RESUMO
O estudo teve como objetivo analisar a comercialização de madeira serrada de espécies nativas do Estado de Mato Grosso, no período de 2004 a 2009, obtendo a classificação das Regiões de Planejamento (RPs) e dos municípios fornecedores de madeira. Os dados foram coletados no posto de fiscalização do Instituto de Defesa Agropecuária do Estado de Mato Grosso (INDEA/ MT), no distrito industrial do município de Cuiabá, onde todos os caminhões que passaram pelo posto transportando madeira foram vistoriados. Por meio da nota fiscal e da guia florestal, foram identificados os municípios fornecedores da madeira e a quantidade transportada. Foi observado que a comercialização de madeira durante o período avaliado apresentou taxa crescente ao decorrer dos anos, porém com taxas decrescentes anuais. Dos 141 municípios do Estado, 81 deles $(54,7 \%)$ participaram como fonte de origem da madeira comercializada, nos quais as RPs XII (Centro-Norte), I (Noroeste I), II (Norte), X (Centro) e XI (Noroeste II) foram as que mais forneceram madeira, respondendo por $92,7 \%$ do volume total comercializado durante o período avaliado.
\end{abstract}

Palavras-chave: Mato Grosso, polos madeireiros, comercialização de madeiras.

\section{Marketing of Wood from Natural Forests in the State of Mato Grosso from 2004 to 2009}

\begin{abstract}
The purpose of this study was to analyze the lumber commercialization of tropical forest native tree species in the State of Mato Grosso from 2004 to 2009, through the classification of the "Planning Regions" and municipalities' suppliers of wood. The data were collected at the checking station of the Institute for Agricultural Defense (INDEA/MT), in the industrial district of Cuiaba, State of Mato Grosso. All trucks which passed through the checkpoint transporting wood were inspected. It was possible to identify the wood municipalities' suppliers and the transported volume through wood load documents such as fiscal receipts and forest guide forms. It was observed that, in the analyzed period, the wood marketing presented the rate increased over the years, but with decreasing annual rates. Out of the 141 municipalities, 81 (54.7\%) were identified as origin sources of the commercialized wood. The Planning Regions XII, I, II, X and XI, were the ones with the greatest contribution $(92.7 \%)$ to the total wood volume commercialized in the analyzed period.
\end{abstract}

Keywords: State of Mato Grosso (MT), timber pole, wood commercialization. 


\section{INTRODUÇÃO}

O território brasileiro encontra-se recoberto pelos mais variados ecossistemas florestais (Floresta Amazônica, Mata Atlântica, Cerrado, Caatinga e outros), o que o posiciona entre os países com maior diversidade do planeta. Além disso, o País conta com plantações nativas e exóticas distribuídas em praticamente todo o território nacional. De acordo com a ABIMCI (Associação..., 2008), o Brasil possui cerca de 550 milhões de hectares de floresta nativa, representando cerca de $10 \%$ de toda a área florestal do mundo. Destes, $60 \%$ são florestas tropicais, $34 \%$ cerrados, $4 \%$ matas de caatinga e $2 \%$ de mata atlântica, sendo que em 2008 o Estado do Amazonas possuía 68,9 milhões de ha, seguido do Pará com 61,9, Mato Grosso com 25,6, Rondônia com 9,9 e, em conjunto, os Estados Acre, Maranhão, Amapá, Roraima e Tocantins, com 45,2, totalizando 211,5 milhões de ha de florestas nativas de produção.

Estima-se que o Setor Florestal no Brasil é responsável por 3,5\% do Produto Interno Bruto-PIB de 2007 do Brasil, equivalente a 37,3 bilhões de dólares, e por $7,3 \%$ das exportações totais do País, equivalente a 10,3 bilhões de dólares, sendo este montante dividido entre os seguintes setores: de celulose, responsável por 4 bilhões de dólares; de madeira serrada, compensados e produtos de maior valor agregado, por 2,9 bilhões de dólares; de móveis, por 1,05 bilhão de dólares, e de carvão vegetal, por 1,65 bilhão de dólares. O Setor Florestal é ainda responsável por gerar cerca de 7 milhões de empregos (Brasil, 2008).

Em relação às exportações brasileiras de produtos de base florestal, os Estados Unidos continuam sendo o principal mercado importador. A grande participação está no segmento de madeira, sendo que, no último ano, as vendas externas a esse país atingiram quase $50 \%$ dos 3,03 bilhões de dólares exportados (Revista..., 2006).Em 2005, as exportações brasileiras de produtos de base florestal tiveram um novo recorde. Com crescimento de $10 \%$ em relação ao ano anterior, as vendas externas de madeira, móveis, papel e celulose atingiram no último ano o volume de 8,21 bilhões de dólares. Os produtos de madeira também tiveram grande desempenho, crescendo $4,2 \%$, em um ano considerado bastante difícil para as indústrias do setor. Com isso, as exportações atingiram 3,15 bilhões de dólares (Revista..., 2007).
Em 2007, mesmo diante de limitações cambiais, as exportações brasileiras de produtos de base florestal apresentaram outro recorde. No conjunto, os segmentos de madeira, móveis, papel e celulose exportaram, em 2007, 9,07 bilhões de dólares, representando um crescimento de $10 \%$ sobre os 8,2 bilhões de dólares no ano anterior. O volume também é expressivo no conjunto das exportações brasileiras, representando cerca de $7 \%$ do total nacional exportado (Revista..., 2008). Já em 2008, o setor madeireiro sentiu os efeitos da crise internacional, quando houve fechamentos de muitas empresas que trabalhavam exclusivamente com exportação. Mato Grosso, ocupando o quinto lugar no ranking de exportações, apresentou uma queda de $50 \%$ no volume de vendas no primeiro trimestre daquele ano: de 55 milhões de dólares negociados, caiu para 25 milhões de dólares (Global 21, 2009).

Após oito anos sucessivos de crescimento, as exportações do setor de base florestal sofreram um duro golpe em 2009. O volume total das vendas externas de madeira, móveis, papel e celulose totalizou 7,38 bilhões de dólares, representando uma queda de $23 \%$ em relação aos 9,58 bilhões de dólares conquistados no ano anterior.

A forte crise mundial, iniciada ainda em 2008, trouxe como relação direta a retração econômica dos principais países consumidores, diminuindo investimentos e, por consequência, as compras internacionais. Das exportações de 2009, a queda mais expressiva foi nos itens de madeira, cujas exportações chegaram a 1,68 bilhão de dólares, volume 39\% menor que o alcançado no mesmo período de 2008, quando atingiu 2,75 bilhões de dólares. Os itens de móveis também tiveram expressiva queda. Em 2009, totalizaram 705,9 milhões de dólares, uma queda de 28,5\% sobre o volume de 2008 (Revista..., 2010).

O maior Estado exportador dos itens de madeira continua sendo o Paraná, com US\$ 531 milhões, vindo a seguir Santa Catarina, com US\$349 milhões, e o Pará, com US\$ 346 milhões. Dos países importadores, os Estados Unidos continuam sendo o principal comprador, com US\$ 497 milhões em 2009, embora esse valor tenha representado uma queda de $36 \%$ em relação ao ano anterior. A França é o segundo principal mercado comprador, com US\$ 126 milhões, mas também com queda de 34\%, e o Reino Unido, com US\$ 102 milhões e uma redução de $36 \%$ em relação ao ano anterior. As maiores 
quedas, porém, vieram de outros importantes países importadores: a Holanda, que importou US\$ 132 milhões em 2008, caiu para US\$ 58 milhões em 2009 (queda de 56\%), e a Espanha, que de US\$ 87 milhões caiu para US\$ 39 milhões (redução de 55\%) (Revista..., 2010).

As florestas naturais são as principais fontes de matéria para as indústrias madeireiras dos Estados, tanto para lenha, quanto para madeiras em toras. A madeira é extraída das florestas mediante a apresentação e a aprovação de planos de manejo florestal sustentado ou de planos de desmatamento. O Estado do Mato Grosso é um importante produtor de madeira em toras, sendo seu volume correspondente a $13,4 \%$ da produção nacional, sendo $20,8 \%$ proveniente da Região Norte e 97,2\% da Região Centro-Oeste. A produção de madeira em toras foi de 2,9 milhões de $\mathrm{m}^{3}$. No entanto, o volume de madeira em toras proveniente de florestas plantadas contribuiu com apenas $0,02 \%$ da produção nacional, sendo $0,5 \%$ proveniente da Região Norte e $1,1 \%$ da Região Centro-Oeste (Passos \& Mason, 2005).

Em 2009, foram identificadas 2.226 empresas madeireiras em funcionamento na Amazônia Legal. Nesse ano, essas madeireiras extraíram em torno de 14,2 milhões de $\mathrm{m}^{3}$ de madeira em tora nativa, o equivalente a 3,5 milhões de árvores. Aproximadamente $47 \%$ dessa matéria-prima foi extraída no Estado do Pará. O volume de madeira em tora extraída em Mato Grosso correspondeu a $28 \%$ do total, enquanto em Rondônia, representou 16\%. O restante (9\%) ocorreu nos Estados de Acre e Amazonas (3\% cada), seguidos de Amapá, Maranhão e Roraima (com cerca de $1 \%$ cada um) (Hummel et al., 2010).

As cidades com o maior número de empresas instaladas dentro do Estado de Mato Grosso, nesse segmento, são Sinop, Marcelândia, Feliz Natal, Juína e Juara. As indústrias de base florestal no Estado compreendem 1.721 empreendimentos, com faturamento bruto anual de 1,9 bilhão de reais, 160 mil empregos diretos e indiretos ligados setor florestal, 210 milhões de dólares de exportação anual, sendo nos anos de 2006, 2007 e 2008 arrecadados 75,3 milhões de reais de ICMS e 12,7 milhões de reais do FETHAB (Consciência..., 2009).

Por sua vez, na Amazônia legal, a indústria madeireira gerou aproximadamente $204 \mathrm{mil}$ empregos, dos quais 66 mil empregos diretos (processamento e exploração florestal) e $137 \mathrm{mil}$ empregos indiretos. Ou seja, em média, cada emprego direto gerou 2,06 postos de trabalho indiretos, nos segmentos de transporte de madeira processada, revenda de madeira processada, lojas de equipamentos e maquinário para o setor madeireiro, consultoria florestal (elaboração de planos de manejo florestal), consultoria jurídica e no beneficiamento da madeira processada para a fabricação de móveis em movelarias (Hummel et al., 2010).

O conhecimento da solidez do mercado de madeira de espécies nativas incentiva a adoção de políticas específicas para a região e para o setor, no sentido de viabilizar os planos de manejo florestal sustentável ou de impacto reduzido (PMFS), e a adoção de mecanismos de desenvolvimento limpo (MDL). Portanto o estudo teve como objetivo analisar a comercialização de madeira serrada de florestas naturais do Estado de Mato Grosso, efetuado via modal rodoviário, no período de 2004 a 2009, identificando os municípios de origem da madeira, caracterizando as regiões do Estado mais representativas em termos de volume comercializado, bem como relatar os prováveis polos madeireiros para o Estado.

\section{MATERIAL E MÉTODOS}

O levantamento da comercialização estadual de madeira, via modal rodoviário, foi realizado no Estado de Mato Grosso no período de 2004 a 2009. O Estado tem uma extensão territorial de $906.806,89 \mathrm{~km}^{2}$, situado entre os paralelos $7^{\circ} 20^{\prime} 30^{\prime \prime}$ e $18^{\circ} 10^{\prime} 00^{\prime \prime}$ latitude Sul e os meridianos $50^{\circ} 13^{\prime} 48^{\prime \prime}$ e $61^{\circ} 31^{\prime} 00^{\prime \prime}$ a Oeste de Greenwich, ocupando uma área equivalente a $10,30 \%$ do território brasileiro. Genericamente, a superfície do Estado é dividida por três áreas distintas, segundo o tipo de vegetação: Pantanal, ocupando aproximadamente $10 \%$ da área territorial; Cerrado, com aproximadamente $40 \%$, e Floresta Amazônica, que cobre cerca de $50 \%$ do território estadual (Ribeiro Junior, 2003).

O Estado tem 141 municípios distribuídos em cinco mesorregiões, 22 microrregiões e 12 Regiões de Planejamento (RP). Para a análise dos dados, utilizou-se a divisão do Estado em 12 Regiões de Planejamento (RP) com os respectivos municípios, 
conforme metodologia adotada pela Secretaria de Estado de Planejamento e Coordenação Geral (Mato Grosso, 2010) (Figura 1).

A metodologia adotada é semelhante à utilizada por Sampaio e Mazzochin (2010), que consistiu em: análise de informações de autores que desenvolveram considerações sobre o tema e leitura de documentos específicos, como relatórios, anuários estatísticos e informações fornecidas pelos institutos e órgãos oficiais do setor de base florestal - IBGE (Instituto Brasileiro de Geografia e Estátistica), ABRAF (Associação Brasileira de Produtores de Florestas Plantadas), ABIMCI (Associação Brasileira da Indústria de Madeira Processada Mecanicamente), entre outros. Dessa forma, os dados não foram coletados apenas por meio de entrevistas.
Os dados foram obtidos no posto de fiscalização rodoviária do Instituto de Defesa Agropecuária do Estado de Mato Grosso (INDEA-MT) no distrito industrial de Cuiabá, durante todos os dias da semana, 24 horas por dia, sem exceção, sendo que todos os caminhões que passaram pelo posto, nesse período, transportando madeira, foram vistoriados. Os dados coletados foram computados em tabelas de acordo com as Regiões de Planejamento, os municípios de origem e o volume comercializado.

As cargas de madeira foram submetidas à rigorosa fiscalização tanto na documentação, na nota fiscal (NF) e na guia florestal (GF3), quanto no volume transportado em metros cúbicos, na medição da carga e na identificação das espécies transportadas, para avaliar a conformidade entre a carga e a respectiva nota fiscal. Para identificação das espécies,

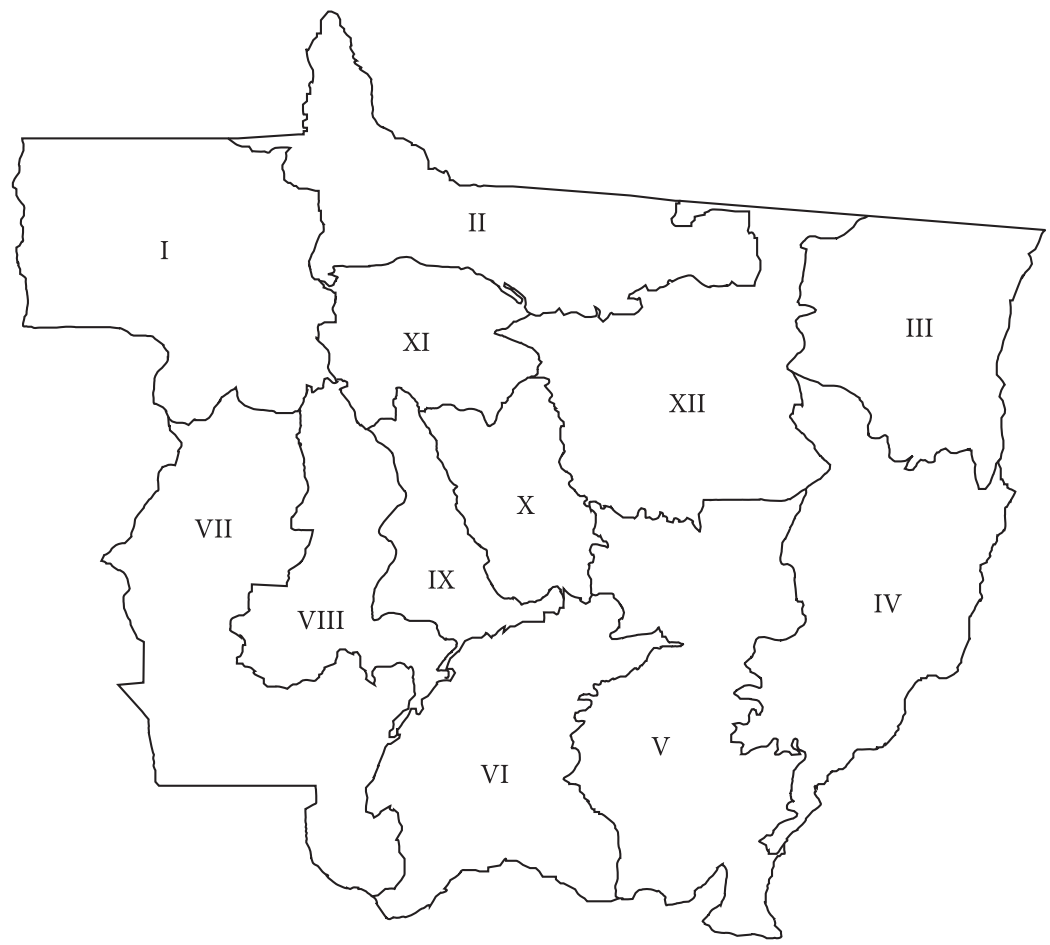

\begin{tabular}{lll}
\hline RP I - Juína - Noroeste & RP II - Alta floresta - Norte & RP III - Vila Rica - Nordeste \\
RP IV - Barra do Garças - Leste & RP V - Rondonópolis - Sudeste & RP VI - Cuiabá/V. Grande - Sul \\
RP VII - Cáceres - Sudoeste & RP VIII - Tangará da Serra - Oeste & RP IX - Diamantino - Centro - Oeste \\
RP X - Sorriso - Centro & RP XI - Juara - Noroeste II & RP XII - Sinop - Centro - Norte
\end{tabular}

Figura 1. Regiões de Planejamento (RP) do Estado de Mato Grosso, propostas pela SEPLAN-MT. Fonte: Mato Grosso (2010).

Figure 1. Planning Regions (RP) of the State of Mato Grosso proposed by SEPLAN-MT. Source: Mato Grosso (2010). 
os fiscais retiravam amostras das madeiras contidas no caminhão e, com auxílio de lupa, identificavam macroscopicamente a espécie transportada. Uma vez verificada a conformidade das informações declaradas com a carga transportada, o INDEA emite o certificado de identificação da madeira. Por meio da nota fiscal (NF) e da guia florestal (GF3), foram identificados os municípios de origem da madeira, as respectivas Regiões de Planejamento e os volumes comercializados.

\section{RESULTADOS E DISCUSSÃO}

Foi constatado que, no período avaliado, os municípios que compõem as Regiões de Planejamento propostas pela SEPLAN (Mato Grosso,
2010) não se constituíram em sua totalidade como municípios fornecedores de madeira, na medida em que alguns municípios que dão nome às referidas Regiões de Planejamento não se apresentaram como fornecedores de madeira. A Tabela 1 apresenta a relação dos municípios do Estado de Mato Grosso, dentro das Regiões de Planejamento, em que ocorreu a comercialização de madeira de mata nativa.

Dentre os 141 municípios que compõem o Estado, apenas 81 comercializaram madeira, o que corresponde a pouco mais da metade $(57,4 \%)$ do total de municípios existentes. Todas as Regiões de Planejamento do Estado, em maior ou menor grau, participaram do comércio de madeira de mata nativa no período avaliado (Figura 2).

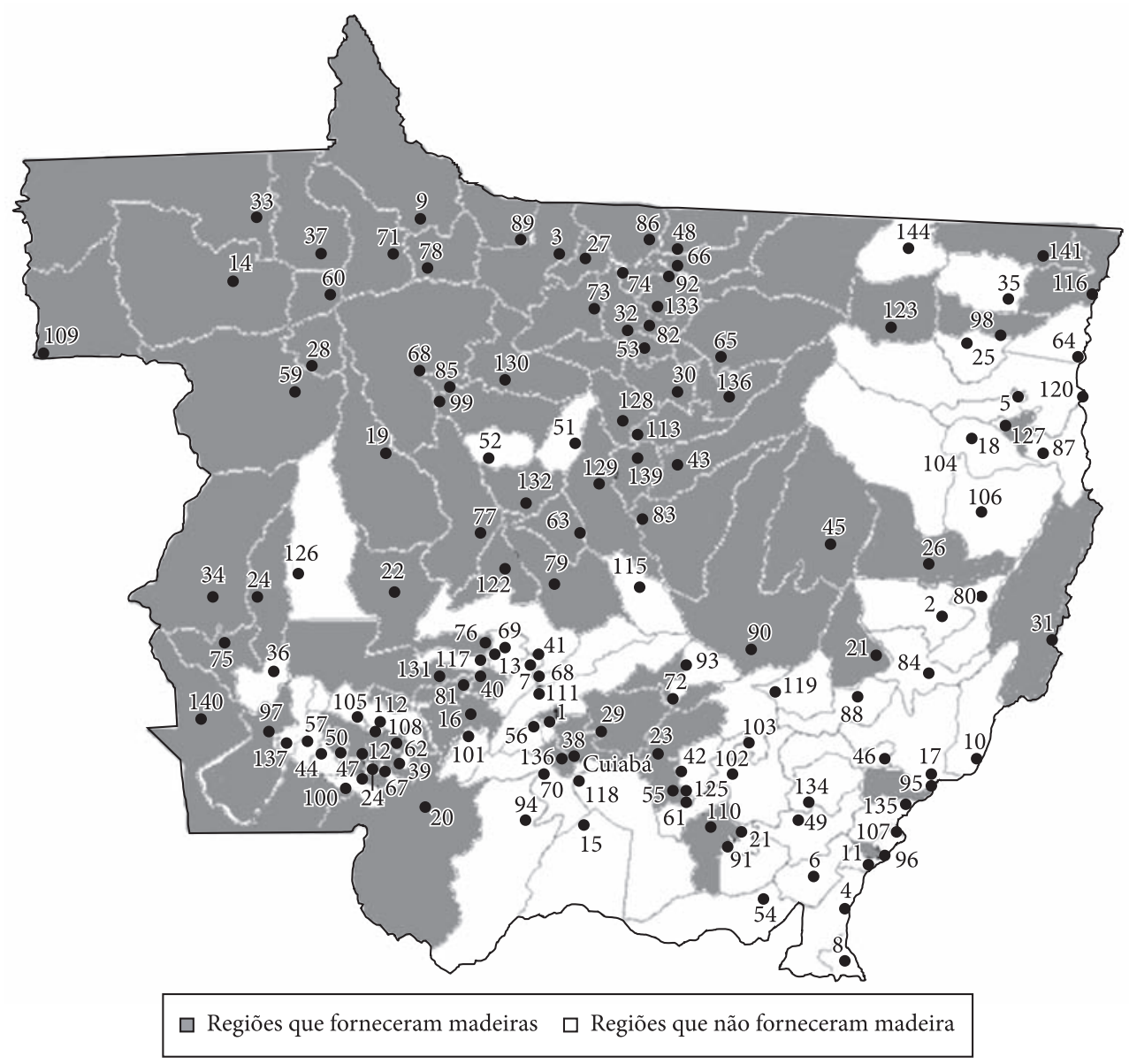

Figura 2. Municípios do Estado de Mato Grosso fornecedores e não fornecedores de madeira de mata nativa comercializada no período de 2004 a 2009.

Figure 2. Municipalities of the State of Mato Grosso suppliers and non-suppliers of native forest commercialized in the period of 2004 to 2009 . 
Tabela 1. Municípios do Estado de Mato Grosso fornecedores de madeira de mata nativa para comercialização no período de 2004 a 2009.

Table 1. Municipalities of the State of Mato Grosso suppliers of native forest to commercialization in the period of 2004 to 2009.

\section{Regióes de planeja- mento (RP)}

\section{Municípios que forneceram madeira}

\begin{tabular}{|c|c|c|}
\hline \multirow{4}{*}{ I-Noroeste I } & 14 - Aripuanã & 59 - Juína \\
\hline & 28 - Castanheira & 60 - Juruena \\
\hline & 37 - Cotriguaçú & 109 - Rondolândia \\
\hline & 33 - Colniza & \\
\hline \multirow{8}{*}{ II-Norte } & 3 - Alta Floresta & 74 - Nova Guarita \\
\hline & 9 - Apiacás & 78 - Nova Monte Verde \\
\hline & 27 - Carlinda & 82 - Nova Santa Helena \\
\hline & 32 - Colíder & 86 - Novo Mundo \\
\hline & 48 - Guarantã do Norte & 89 - Paranaíta \\
\hline & 66 - Matupá & 92 - Peixoto de Azevedo \\
\hline & 71 - Nova Bandeirantes & 133 - Terra Nova do Norte \\
\hline & 73 - Nova Canaã do Norte & \\
\hline \multirow{2}{*}{ III-Nordeste } & 98 - Porto Alegre do Norte & 123 - São José do Xingu \\
\hline & 116 - Santa Terezinha & 127 - Serra Nova Dourada \\
\hline \multirow{3}{*}{ IV-Leste } & 21 - Campinápolis & 95 - Pontal do Araguaia \\
\hline & 26 - Canarana & 96 - Ponte Branca \\
\hline & 31 - Cocalinho & \\
\hline \multirow{3}{*}{ V-Sudeste } & 23 - Campo Verde & 90 - Paranatinga \\
\hline & 45 - Gaúcha do Norte & 110 - Rondonópolis \\
\hline & 55 - Jaciara & \\
\hline \multirow{3}{*}{ VI-Sul } & 1 - Acorizal & 72 - Nova Brasilândia \\
\hline & 29 - C. dos Guimarães & 138 - Várzea Grande \\
\hline & 38 - Cuiabá & \\
\hline \multirow{7}{*}{ VII-Sudoeste } & 12 - Araputanga & 62 - Lambari d'Oeste \\
\hline & 20 - Cáceres & 67 - Mirassol d'Oeste \\
\hline & 24 - Campos de Júlio & 75 - Nova Lacerda \\
\hline & 34 - Comodoro & 97 - Pontes e Lacerda \\
\hline & 39 - Curvelândia & 100 - Porto Esperidião \\
\hline & 50 - Indiavaí & 108 - Rio Branco \\
\hline & 57 - Jaurú & 140 - Vila Bela da Santíssima Trindade \\
\hline \multirow{3}{*}{ VIII-Oeste } & 16 - Barra do Bugres & 40 - Denise \\
\hline & 19 - Brasnorte & 81 - Nova Olímpia \\
\hline & 22 - Campo Novo do Parecis & 131 - Tangará da Serra \\
\hline \multirow{2}{*}{ IX - Centro-Oeste } & 76 - Nova Marilândia & 122 - São José do Rio Claro \\
\hline & 77 - Nova Maringá & \\
\hline \multirow{2}{*}{ RP X-Centro } & 63 - Lucas do Rio Verde & 129 - Sorriso \\
\hline & 79 - Nova Mutum & 132 - Tapurah \\
\hline \multirow{2}{*}{ XI-Noroeste II } & 58 - Juara & 99 - Porto dos Gaúchos \\
\hline & 85 - Novo Horizonte do Norte & 130 - Tabaporã \\
\hline \multirow{5}{*}{ XII-Centro -norte } & 30 - Cláudia & 113 - Santa Carmen \\
\hline & 43 - Feliz Natal & $128-$ Sinop \\
\hline & 53 - Itaúba & 136 - União do Sul \\
\hline & 65 - Marcelândia & 139 - Vera \\
\hline & 83 - Nova Ubiratã & \\
\hline
\end{tabular}


As Regiões de Planejamento XII, II, I, XI e X foram as mais representativas, contribuindo com 92,73\% do volume total de madeira comercializada nos anos avaliados. Na medida em que essas regiões foram as que forneceram maiores quantidade de madeira em metros cúbicos, é de se esperar que o(s) polo(s) madeireiro(s) do Estado de Mato Grosso esteja(m) localizado(s) em algum dos municípios dessas regiões. As demais regiões corresponderam a 7,27\% do volume total comercializado. (Tabela 2).

A Região de Planejamento XII (Centro-Norte) foi a mais importante em termos de volume comercializado, com $38,15 \%$ do total, sendo o município de Sinop o que gerou maior fornecimento de madeira. Segundo Redivo et al. (2010), no município de Sinop, desde a década de 1980, vêm crescendo o extrativismo e o beneficiamento de madeiras, fazendo com que, atualmente, o município seja considerado um grande polo madeireiro do Estado do Mato Grosso. As Regiões de Planejamento VI, V, III e IV foram as que forneceram os menores volumes de madeira, contribuindo, conjuntamente, com menos de $1 \%$ do total.

Comparando-se o percentual dos dados referentes ao volume anual em metros cúbicos de madeira de mata nativa comercializada pelo Estado, pôde-se perceber que em 2004 foram comercializados 403.827,85 $\mathrm{m}^{3}$ e, em 2005, $319.786,89 \mathrm{~m}^{3}$, com um decréscimo de 20,81\% no volume de madeira de um ano para o outro. A queda de $20 \%$ no volume comercializado no ano de 2005 em relação a 2004, conforme se observa na Tabela 3, deve-se à realização das Operações Curupira I e II, desencadeadas, respectivamente, em Junho e Agosto

Tabela 2. Volume total comercializado, por região de planejamento, em ordem decrescente, no período de 2004 a 2009.

Table 2. Total volume commercialized, by region planning, in descending order in the period of 2004 to 2009.

\begin{tabular}{|ccccc|}
\hline Ordem & RP & Localização & Volume total $\left(\mathbf{m}^{3}\right)$ & $\%$ \\
\hline $1^{\circ}$ & XII & Centro-Norte & $1.881 .090,77$ & 38,15 \\
$2^{\circ}$ & I & Noroeste I & $920.973,06$ & 18,67 \\
$3^{\circ}$ & II & Norte & $644.433,15$ & 13,10 \\
\hline $4^{\circ}$ & X & Centro & $586.330,71$ & 11,89 \\
\hline $5^{\circ}$ & XI & Noroeste II & $538.785,04$ & 10,92 \\
$6^{\circ}$ & VIII & Oeste & $184.922,80$ & 3,75 \\
\hline $7^{\circ}$ & IX & Centro-Oeste & $101.803,09$ & 2,06 \\
$8^{\circ}$ & VII & Sudoeste & $53.877,13$ & 1,09 \\
\hline $9^{\circ}$ & VI & Sul & $15.370,84$ & 0,32 \\
\hline $10^{\circ}$ & V & Sudeste & $1.838,46$ & 0,03 \\
\hline $11^{\circ}$ & III & Nordeste & $1.348,47$ & 0,02 \\
\hline $12^{\circ}$ & IV & Leste & 147,16 & 0,01 \\
\hline
\end{tabular}

Tabela 3. Variação e volume anual de madeira de mata nativa comercializada pelo Estado de Mato Grosso, no período de 2004 a 2009.

Table 3. Variation and annual volume of native forest commercialized by State of Mato Grosso, in the period of 2004 to 2009 .

\begin{tabular}{ccccc}
\multirow{2}{*}{ Ano } & \multicolumn{2}{c}{ Volume } & \multicolumn{2}{c}{ Variação anual } \\
\cline { 2 - 4 } \cline { 5 - 5 }$\left(\mathbf{m}^{3}\right)$ & $(\%)$ & - & $(\%)$ \\
\hline 2004 & $403.827,85$ & 8,18 & $(-) 84.040,96$ & - \\
\hline 2005 & $319.786,89$ & 6,50 & $214.944,42$ & 67,21 \\
\hline 2006 & $534.731,31$ & 10,84 & $316.082,50$ & 59,11 \\
\hline 2007 & $850.813,81$ & 17,25 & $388.083,71$ & 45,61 \\
\hline 2008 & $1.238 .897,52$ & 25,12 & $343.965,82$ & 27,76 \\
\hline Média & $1.582 .863,34$ & 32,10 & $235.807,09$ & 35,77 \\
\hline
\end{tabular}


de 2005 pela Policia Federal e pelo Ministério do Meio Ambiente, as quais atingiram diretamente o transporte rodoviário de madeira no Estado de Mato Grosso.

Essas informações são confirmadas por Moura (2006), destacando que, no período de Agosto de 2003 a Agosto de 2004, o Estado de Mato Grosso sozinho foi responsável por $50 \%$ do desmatamento ocorrido em toda a Floresta Amazônica, constituindo-se no foco principal das Operações Curupira I e II. A ocorrência dessas operações foi em razão de suspeitas de procedimentos ilegais e corrupção na emissão e no uso das ATPFs (Autorizações de Transportes de Produtos Florestais).

O volume total de madeira comercializada durante o período avaliado foi de 4.930.920,68 $\mathrm{m}^{3}$, com um volume médio anual de 821.815,63 $\mathrm{m}^{3}$; note-se que a partir de 2005 houve um acréscimo considerável, superior a 2004, mantendo-se crescente nos demais anos e culminando com uma produção de $1.582 .863,34 \mathrm{~m}^{3}$ em 2009, a qual representou um crescimento de $495 \%$ do volume comercializado no período avaliado. Uma hipótese que pode explicar esse aumento seria o fato de que, após as operações da Polícia Federal, as fiscalizações aumentaram nos anos seguintes, fazendo com que aumentasse a quantidade de madeira comercializada legalmente. De acordo com Brasil 2010, o fornecimento de madeira tende a crescer com o passar dos anos, pois cada vez mais a demanda de madeira vem aumentando e tende a continuar crescendo, o que explica o aumento do fornecimento de madeira para o mercado.

No ano de 2009, foi comercializado $32,1 \%$ do volume total de madeira do período, seguido de 2008 com $25,12 \%$ e 2007 com $17,25 \%$, enquanto conjuntamente 2004, 2005 e 2006 foram responsáveis por $25,52 \%$ da produção total do período, evidenciando que, em 2007, 2008 e 2009, cerca de $75 \%$ do volume total de madeira foi comercializado via modal rodoviário no Estado de Mato Grosso. De acordo com Pereira et al. (2010), em 2009, os 71 polos processadores de madeira da Amazônia Legal extraíram aproximadamente 14,2 milhões de $\mathrm{m}^{3}$ de madeira em tora e o Estado de Mato Grosso foi o segundo maior produtor, respondendo por $32 \%$ do total produzido e ficando atrás do Pará, que respondeu por $44 \%$.
A RP XII (Região Centro-Norte) forneceu mais de $100 \mathrm{mil} \mathrm{m}^{3}$ anuais ao longo do período estudado, ou seja, reuniu características fundamentais para a localização do principal polo madeireiro do Estado de Mato Grosso, pois, conforme Veríssimo et al. (2002), polo madeireiro é um município ou uma região que fornece anualmente pelo menos $100 \mathrm{mil} \mathrm{m}^{3} \mathrm{de}$ madeira em tora em processos industriais. No período de 2007 a 2009, as RPs II e XI (Norte e Noroeste II, respectivamente) aumentaram o fornecimento de madeira, contribuindo com mais de $100 \mathrm{mil} \mathrm{m}^{3} \mathrm{em}$ relação aos anos anteriores (Tabela 4), se mostrando também como polos madeireiros. Na Região XI, está situado o município de Juara e, de acordo com a Sema (2008), o município apresenta-se como um produtor de toras em volume, também apresentando maior diversidade de espécies exploradas - 155 no total, sendo que 79 são encontradas apenas nesse município.

A RP XII permaneceu em primeiro lugar na classificação anual por volume de madeira comercializado, em todos os anos estudados, com uma média de $38,15 \%$ do volume total comercializado. Pode ser observada uma alternância ao longo dos 6 anos na ocupação do segundo lugar na classificação, ficando RP I (Região Noroeste I-Juína) nos anos de 2004 e 2005, RP XI (Região Noroeste IIJuara) em 2006 e 2007, sendo que, em 2008 e 2009, a RP I volta a ocupar essa posição. Em terceiro lugar, ficou a RP XI (Região Noroeste II-Juara) no ano de 2004, RP II (Região Norte-Alta Floresta) nos anos de 2005, 2006, 2007 e 2008, sendo essa posição, em 2009, ocupada pela RP X (Região Centro-Sorriso) (Tabela 5).

Do sexto ao $12^{\circ}$ lugar, houve movimentação de madeira em quantidades irrisórias em termos relativos, em que se enquadram, em ordem decrescente de volume comercializado, as RP VIII (Região Oeste), RP IX (Região Centro-Oeste), RP VII (Região Sudeste), RP VI (Região Sul), RP V (Região Sudeste), RP III (Região Nordeste) e RP IV (Região Leste). No conjunto, essas sete regiões respondem por $359.307,95 \mathrm{~m}^{3}$ comercializados nos seis anos, ou $7,27 \%$ do volume total (menos de $5.000 \mathrm{~m}^{3}$ por mês), não se caracterizando como um dos prováveis polos madeireiros do Estado de Mato Grosso, tendo-se por base o volume de madeira. 
Tabela 4. Volume total de madeira de mata nativa comercializada pelo Estado de Mato Grosso, por região de planejamento, no período de 2004 a 2009.

Table 4. Volume of native forest commercialized by State of Mato Grosso, by planning regions in the period of 2004 to 2009 .

\begin{tabular}{|crrrrrr|}
\hline \multirow{2}{*}{ RP } & \multicolumn{7}{c|}{ Ano } \\
\cline { 2 - 7 } & $\mathbf{2 0 0 4}$ & $\mathbf{2 0 0 5}$ & $\mathbf{2 0 0 6}$ & $\mathbf{2 0 0 7}$ & \multicolumn{1}{c|}{$\mathbf{2 0 0 8}$} & $\mathbf{2 0 0 9}$ \\
\hline I & $56.891,35$ & $57.066,83$ & $67.736,05$ & $89.713,15$ & $247.694,67$ & $401.871,00$ \\
\hline II & $52.066,60$ & $48.468,39$ & $75.936,85$ & $118.725,31$ & $224.843,72$ & $124.392,26$ \\
\hline III & 25,02 & 934,55 & 60,14 & 84,83 & 177,58 & 66,34 \\
\hline IV & 42,46 & 104,70 & 0,00 & 0,00 & 0,00 & 0,00 \\
\hline V & 32,14 & $1.484,44$ & 47,03 & 63,08 & 126,65 & 85,10 \\
\hline VI & $1.525,25$ & $3.811,68$ & $3.826,15$ & 372,32 & $5.641,28$ & 194,15 \\
\hline VII & $21.800,85$ & $16.725,59$ & $6.527,37$ & $4.570,03$ & $2.796,94$ & $1.456,33$ \\
\hline VIII & $12.791,35$ & $4.764,36$ & $12.316,09$ & $26.339,90$ & $61.586,79$ & $67.124,31$ \\
\hline IX & $21.191,45$ & $7.916,55$ & $14.705,27$ & $15.782,03$ & $9.294,90$ & $32.912,87$ \\
\hline X & $28.361,81$ & $26.270,12$ & $38.035,65$ & $79.359,10$ & $67.072,34$ & $347.231,67$ \\
\hline XI & $53.413,00$ & $42.438,79$ & $91.432,10$ & $135.318,52$ & $110.038,87$ & $106.143,73$ \\
\hline XII & $155.686,50$ & $109.800,85$ & $224.108,58$ & $380.485,49$ & $509.623,75$ & $501.385,58$ \\
\hline
\end{tabular}

Tabela 5. Classificação anual das regiões de planejamento (RP) do Estado de Mato Grosso em volume comercializado no período de 2004 a 2009.

Table 5. Annual ranking of the planning regions of the State of Mato Grosso in volume commercialized in the period of 2004 and 2009.

\begin{tabular}{|c|c|c|c|c|c|c|}
\hline \multirow{3}{*}{ Classificação } & \multicolumn{6}{|c|}{ Ano } \\
\hline & 2004 & 2005 & 2006 & 2007 & 2008 & 2009 \\
\hline & RP & RP & RP & RP & $\mathbf{R P}$ & RP \\
\hline $1^{\circ}$ & XII & XII & XII & XII & XII & XII \\
\hline $2^{\circ}$ & I & I & XI & XI & I & I \\
\hline $3^{\circ}$ & XI & II & II & II & II & $\mathrm{X}$ \\
\hline $4^{\circ}$ & II & XI & I & I & XI & II \\
\hline $5^{\circ}$ & $\mathrm{X}$ & $\mathrm{X}$ & $\mathrm{X}$ & $\mathrm{X}$ & $\mathrm{X}$ & XI \\
\hline $6^{\circ}$ & VII & VII & IX & VIII & VIII & VIII \\
\hline $7^{\circ}$ & IX & IX & VIII & IX & IX & IX \\
\hline $8^{\circ}$ & VIII & VIII & VII & VII & VI & VII \\
\hline $9^{\circ}$ & VI & VI & VI & VI & VII & VI \\
\hline $10^{\circ}$ & IV & V & III & III & III & V \\
\hline $11^{\circ}$ & V & III & V & V & V & III \\
\hline $12^{\circ}$ & III & IV & IV & IV & IV & IV \\
\hline
\end{tabular}

\section{CONCLUSÕES}

O Estado de Mato Grosso comercializou um total de 4.930.920,68 $\mathrm{m}^{3}$ de madeira serrada de mata nativa via modal rodoviário no período de 2004 a 2009, representando um crescimento de $495 \%$ no período, com uma média anual de $821.820,11 \mathrm{~m}^{3} \mathrm{e}$ um crescimento médio de $35,77 \%$ ao ano.
O volume anual comercializado apresentou uma tendência crescente no período; porém, no ano de 2005, houve uma redução do volume comercializado, em decorrência da Operação Curupira, que promoveu uma redução de mais de $20 \%$ no volume comercializado em relação ao ano anterior.

A partir de 2006, o volume anual comercializado cresceu continuamente, porém a taxas decrescentes 
anuais, indicando tendência de redução crescente e continuada do desmatamento na região.

Todas as Regiões de Planejamento do Estado apresentam municípios participando do comércio de madeira serrada de mata nativa no período avaliado, sendo que os municípios das Regiões Centro-Norte e Noroeste do Estado respondem por cerca de $70 \%$ do volume de madeira no período.

A RP XII (Centro-Norte), com 38,15\% do volume total, foi a mais importante Região de Planejamento em termos de volume de madeira serrada de mata nativa comercializada, caracterizando-se como polo madeireiro do Estado.

\section{STATUS DA SUBMISSÃO}

Recebido: 09/08/2011

Aceito: 27/09/2011

Resumo publicado online: 29/09/2011

Artigo completo publicado: 22/12/2011

\section{AUTOR(ES) PARA CORRESPONDÊNCIA}

\section{Edilene Silva Ribeiro}

Programa de Pós-Graduação em Ciências Florestais e Ambientais,

Faculdade de Engenharia Florestal - FENF, Universidade Federal de Mato Grosso - UFMT, Av. Fernando Corrêa da Costa, 2367,

Boa Esperança, CEP 78125-070,

Cuiabá, MT, Brasil

e-mail: eng.edilene@gmail.com

\section{Roberto Antonio Ticle de Melo e Sousa}

Departamento de Engenharia Florestal, Faculdade de Engenharia Florestal - FENF, Universidade Federal de Mato Grosso - UFMT, Av. Fernando Corrêa da Costa, 2367, Boa Esperança, CEP 78125-070, Cuiabá, MT, Brasil,

e-mail: ratms@terra.com.br

\section{REFERENNCIAS}

Associação Brasileira da Indústria de Madeira Processada Mecanicamente-ABIMCI. Estudo Setorial [online]. Curitiba: ABIMCI; 2008. [cited 2011 ago. 23]. Available from: http://www.abimci.com.br/ dmdocuments/ABIMCI_Estudo_Setorial_2008.pdf.

Brasil. Ministério do Meio Ambiente-MMA. As Florestas Plantadas [online]. Brasília: MMA; 2010. [cited 2011 ago. 23]. Available from: http://www.florestal.gov.br/ snif/recursos-florestais/as-florestas-plantadas.

Brasil. Ministério do Meio Ambiente-MMA. Serviço florestal brasileiro [online]. Brasília: MMA; 2008. [cited 2011 ago. 23]. Available from: http://www. florestal.gov.br/snif/producao-florestal/cadeiaprodutiva.

Centro das Indústrias Produtoras e Exportadoras de Madeira do Estado de Mato Grosso - CIPEM. Consciência de uma floresta viva e sustentável [online]. CIPEM; 2009. 7 p. Informativo CIPEM. [cited 2009 nov. 08]. Available from: www.cipem.org.br/novo/arqui vos/812563e3e0b885c1ffa18995ea54d5c2.pdf.

Global 21. Madeira-Setor madeireiro apresenta queda de 50\% no volume de exportações [online]. Expresso MT; 2009. [cited 2011 ago. 22]. Available from: http://www.global21.com.br/materias/materia. asp $? \operatorname{cod}=24796 \&$ tipo $=$ noticia.

Hummel AC, Alves MVS, Pereira D, Veríssimo A, Santos D. A atividade madeireira na Amazônia brasileira: produção, receita e mercados. Belém: IMAZON, 2010. 32 p.

Mato Grosso (Estado). Secretaria de Estado do Meio Ambiente-SEMA. GF1 por município [online]. Cuiabá: SEMA; 2008. [cited 2011 ago. 22]. Available from: http://www.sema.mt.gov.br/relatoriosccsema/.

Mato Grosso (Estado). Secretaria de Estado de Planejamento e Coordenação Geral - SEPLAN. Localização e divisão política de Mato Grosso [online]. Cuiabá: SEPLAN; 2010. 19 p. [cited 2011 ago. 22]. Available from: http://www.indicador.seplan.mt.gov.br/ mtemnumeros2010/pdf/localizacao.pdf.

Moura DG. Mídia e corrupção: a Operação Curupira na Amazônia [Dissertação]. Brasília: Mestrado em Desenvolvimento Sustentável, Universidade de Brasília; 2006.

Passos CAM, Mason R. Potencial Madeireiro do Estado de Mato Grosso. Várzea Grande: CIPEM; 2005. 69 p.

Pereira D, Santos D, Vedoveto M, Guimarães J, Veríssimo A. Fatos Florestais da Amazônia 2010. Belém: IMAZON, 2010. 121 p.

Redivo AR, Redivo A, Sornberger GP. Critérios de qualidade para o mercado nacional e internacional da madeira: um estudo em empresas da micro região de Sinop/MT. Ingepro 2010; 5(2):39-50.

Revista da Madeira-REMADE. Panorama do Comércio Exterior Brasileiro 2005 [online]. Revista da Madeira 2006; (96). [cited 2011 ago. 22]. Available from: 
http://www.remade.com.br/br/revistadamadeira_capa. php? edicao $=96 \&$ button $2=\mathrm{OK}$.

Revista da Madeira-REMADE. Recorde de U\$ $\$ 8,2$ Bilhões em exportações [online]. Revista da Madeira 2007; (102). [cited 2011 ago. 22]. Available from: http://www.remade.com.br/br/revistadamadeira_capa. php?edicao $=102 \&$ button $2=\mathrm{OK}$.

Revista da Madeira-REMADE. Exportações de R\$\$ 9 Bilhões Batem Recorde [online]. Revista da Madeira 2008; (111). [cited 2011 ago. 22]. Available from: http://www.remade.com.br/br/revistadamadeira_capa. php?edicao $=111$ \&button $2=\mathrm{OK}$.

Revista da Madeira-REMADE. Exportações mostram um ano de dificuldades [online]. Revista da
Madeira 2010; (123). [cited 2011 ago. 22]. Available from: http://www.remade.com.br/br/revistadamadeira_capa. php?edicao $=123 \&$ button $2=\mathrm{OK}$.

Ribeiro Junior CVT. Análise da Economia de Exportação do Setor Madeireiro do Estado de Mato Grosso Pós-Plano Real [monografia]. Cuiabá: Graduação em Engenharia Florestal, Universidade Federal de Mato Grosso; 2003.

Sampaio SF, Mazzochin MS. Espacialidade da economia: inovação e estratégias espaciais no setor de base florestal brasileiro. RA'EGA, Curitiba 2010; 20(1):53-65.

Veríssimo A, Lima E, Lentini M. Pólos Madeireiros do estado do Pará. Belém: Instituto do Homem e Meio Ambiente da Amazônia-Imazon. 2002. 72 p. 